\title{
Uterine Arteriovenous Malformations: Therapeutic Strategies and the Place of Hysteroscopy
}

\author{
S. Haddout ${ }^{1}$, A. Agman ${ }^{2}$, P.-F. Ceccaldi ${ }^{3}$ \\ ${ }^{1}$ Assistance Publique des hôpitaux de Paris, Paris, France \\ ${ }^{2}$ Hôpital Beaujon, Assistance Publique des hôpitaux de Paris, Paris, France \\ ${ }^{3}$ Obstétric and Gynecology Department, Hôpital Beaujon, Assistance Publique des hôpitaux de Paris, Paris, France \\ Email: sana.haddout@gmail.com
}

How to cite this paper: Haddout, S., Agman, A. and Ceccaldi, P.-F. (2021) Uterine Arteriovenous Malformations: Therapeutic Strategies and the Place of Hysteroscopy. Open Journal of Obstetrics and Gynecology, 11, 1565-1580.

https://doi.org/10.4236/ojog.2021.1111146

Received: October 4, 2021

Accepted: November 20, 2021

Published: November 23, 2021

Copyright $\odot 2021$ by author(s) and Scientific Research Publishing Inc. This work is licensed under the Creative Commons Attribution International License (CC BY 4.0).

http://creativecommons.org/licenses/by/4.0/

\begin{abstract}
Uterine arteriovenous malformations (UAVMs) are not common and are not well known. They are mainly manifested by hemorrhages that can be life-threatening. Their diagnosis is based mainly on ultrasound coupled with Doppler flow, as well as pelvic magnetic resonance imaging and angiography. In the absence of a consensus, and based on our experience, we discuss the place of hysteroscopy in the therapeutic strategy of UAVM. We reported 3 cases of UAVM, and we performed a review of the literature concerning the diagnosis and treatment of this pathology. We finally collected the advantages of the embolization-hysteroscopy sequence. In fact, the data suggest that hysteroscopy is a feasible and safe alternative treatment modality for UAVM. We believe that the resection of UAVM can be done under optimal conditions with a reduced risk of bleeding. Hysteroscopy would allow for optimal evaluation of the uterine cavity after treatment as well as the possibility of applying an anti-adhesive gel. In addition, the combination of hysteroscopy and embolization would require less healing time and shorter follow-up than embolization alone. It could also reduce the number of patients lost to follow-up.
\end{abstract}

\section{Keywords}

Uterine Arteriovenous Malformation, Hysteroscopy and UAVM,

Hysteroscopy and Trophoblastic Retention

\section{Introduction}

Uterine arteriovenous malformations (UAVM) are defined by the presence of anastomoses between the arteries and veins of the myometrium. Nearly one 
hundred cases have been described in the literature. They are generally acquired. They are often secondary to retention of trophoblastic tissue, but can also follow endo-uterine trauma (curettage, caesarean section or uterus). More rarely, these UAVM are congenital and result from a disorder of embryological vascular differentiation leading to multiple vascular connections. They vary in size and location and occur mainly in women of childbearing age. They often manifest as recurrent and intermittent bleeding. Their sudden onset and abundance can be life-threatening. The diagnosis is usually made by endovaginal ultrasound with Doppler flow. Pelvic MRI and angiography can refine the diagnosis and clarify the vascular afferents.

In the absence of consensus, the treatments described are based on surveillance, embolization and surgery. On the basis of our experience, we asked ourselves the question of the place of hysteroscopy in the therapeutic strategy of UAVM. In this work, we base ourselves on 3 described cases of UAVM that occurred at the Beaujon Hospital, the first of which led to a change in the management of the next two. We will then rely on a review of the literature and discuss the relevance of our therapeutic sequence.

\section{About 3 Cases}

\section{Case $\mathrm{n}^{\circ} 1$}

Mrs CHA. 32 years old, G1P1, underwent a medical termination of pregnancy at $22 \mathrm{WG}$ and 5 days, in a context of chorioamnionitis on premature rupture of membranes at $22 W G+4$. The file does not report uterine revision or emptiness ultrasound. Pathological analysis of the placenta found an incomplete normotrophic placenta with chorioamnionitis lesion without associated funiculitis. One month later, the patient consults for persistent bleeding. Ultrasound finds a heterogeneous vascularized lesion of $4 \mathrm{~cm}$ suggesting retention or UAVM (Figures $1-3)$.

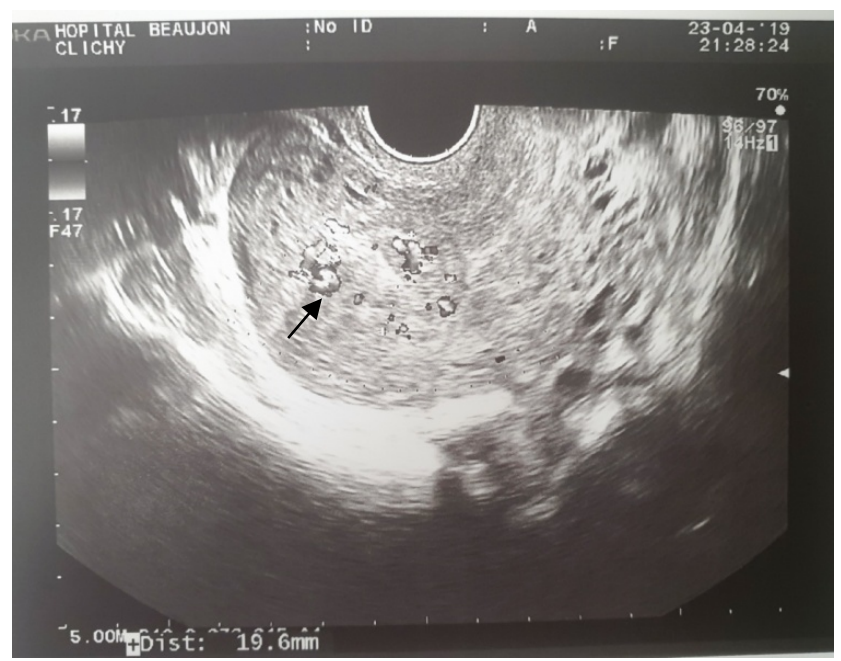

Figure 1. Ultrasound performed in the gynecological emergency room showing a suspicion of UAVM: vascularized peloton of $40 \times 19.6 \mathrm{~mm}(\rightarrow)\left(\right.$ case $\left.\mathrm{n}^{\circ} 1\right)$. 


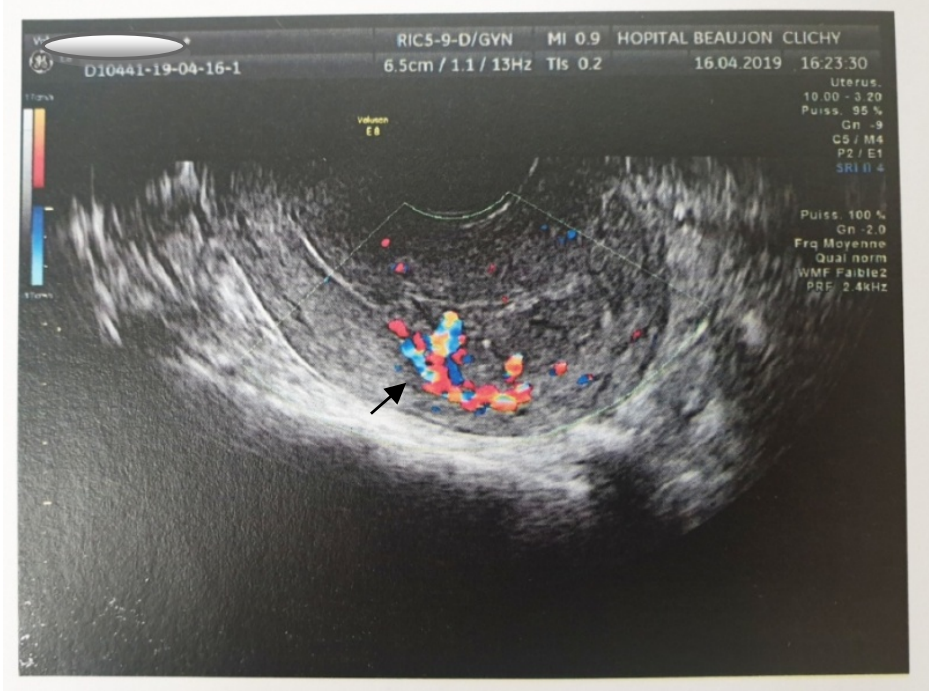

Figure 2. Doppler-coupled ultrasound appearance of UAVM at the posterior corporeal level $(\rightarrow)\left(\right.$ Case $\left.^{\circ} 1\right)$.

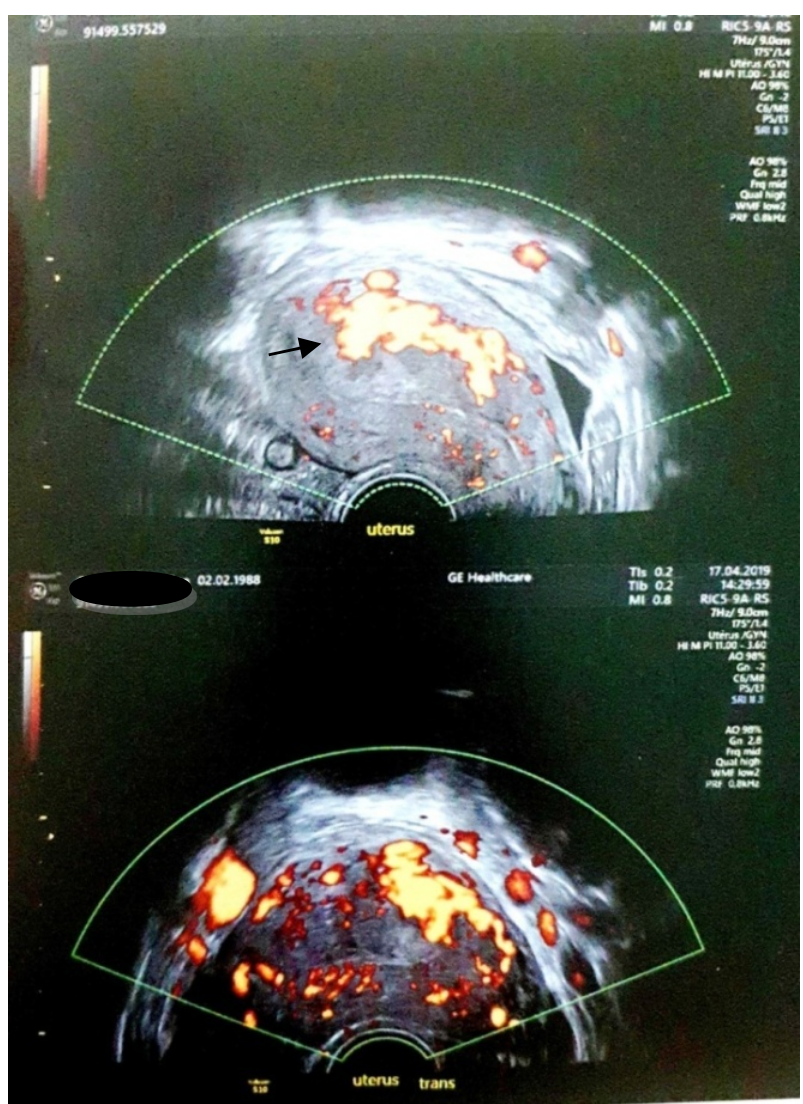

Figure 3. Two ultrasound images showing an image of UAVM enhancing with color Doppler $(\rightarrow)\left(\right.$ Case $\left.^{\circ} 1\right)$.

Hysteroscopy decision for late post-abortion retention. At hysteroscopy we notice a highly vascularized right posterolateral placental retention. Attempted resection of the retained placenta with the loop cut, heavy active bleeding in the 
process. Immediate cessation of hysteroscopy. The bleeding is estimated to be over $1000 \mathrm{~cm}^{3}$. Placement of an endo-uterine Foley catheter swollen to $50 \mathrm{~cm}^{3}$ allowing active bleeding to stop. Withdrawal of the Foley catheter after $20 \mathrm{~min}$ of tamponade, and no resumption of bleeding over time. The minimal debris removed is sent to pathology, which responds: debris from placental retention.

One month later, persistence of small episodes of brownish metrorrhagia without return of the layers proper. On ultrasound, we notice the persistence of a stable vascularized endo-uterine image of $4 \mathrm{~cm}$. Monthly follow-up by ultrasound and pelvic MRI. The patient developed a major depressive syndrome. After 4 months of monitoring, we decide to carry out an embolization, but the patient has a return of the rules and a complete spontaneous regression of the endo-uterine image (Figure 4: Resumption of regular cycles). The patient is pregnant again with a normal course of the pregnancy without any abnormalities in the insertion of the placenta. She gave birth at 39 weeks with a single birth follow-up.

\section{Case $n^{\circ} 2$}

Mrs USE. 22-year-old G3P1 (uncomplicated spontaneous miscarriage, vaginal delivery and surgical suction abortion at 13 weeks +4 days), presented with metrorrhagia 5 weeks after termination of pregnancy, with intermittent bleeding for 2 to 3 days then heavy bleeding. The patient sees the emergency room for bleeding

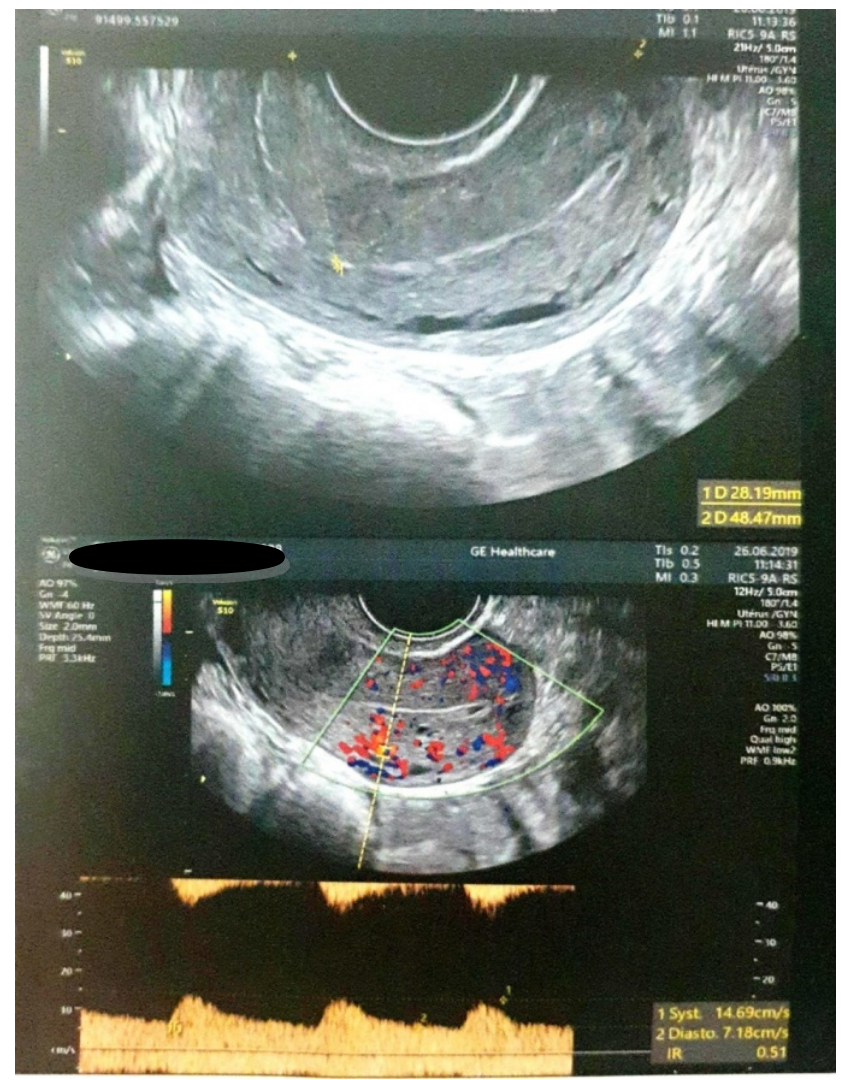

Figure 4. Control ultrasound performed 2 months after hysteroscopy with disappearance of the UAVM (Case $\left.\mathrm{n}^{\circ} 1\right)$. 
and pelvic pain. The endovaginal ultrasound reveals an image that may correspond to an intrauterine retention of $34 \times 39 \times 31 \mathrm{~mm}$. The post-aspiration image showing uterine void, we suspect UAVM or necrobiosis of a posterior submucosal fibroma type 0 or 1 (Figures 5-7), Pelvic MRI performed reveals UAVM (Figure 8 \& Figure 9).

The therapeutic strategy therefore consists of performing a first embolization and a second operative hysteroscopy. Angiography confirms left uterine arterial proliferation without early venous return (Figure 10). The free flow embolization of this artery is performed with absorbable material until complete stasis (GELITASPON). Failure of the catheterization of the right uterine artery whose angle is too sharp and decision to stop the operation for non-participation of the right uterus.

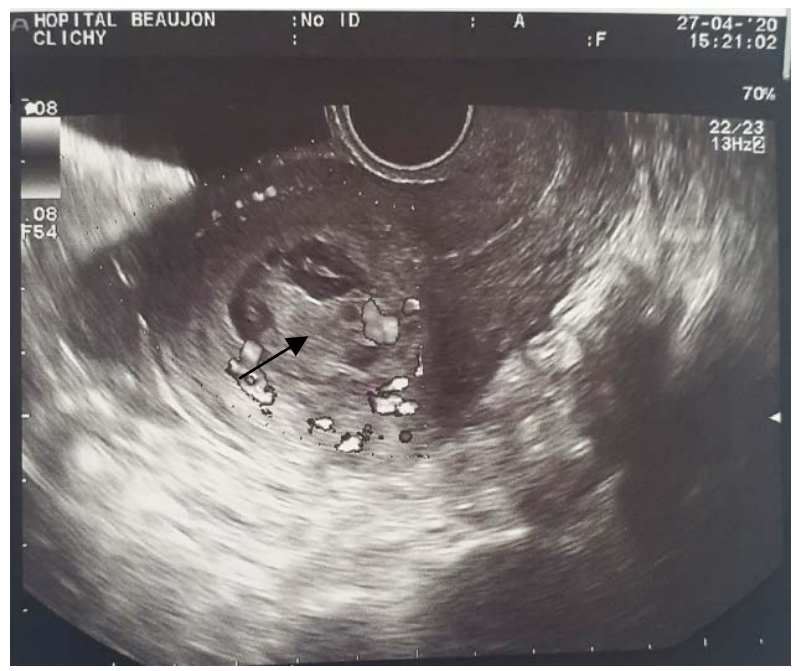

Figure 5. Appearance of necrotic submucosal fibroid or arteriovenous malformation on endovaginal ultrasound $(\rightarrow)$.

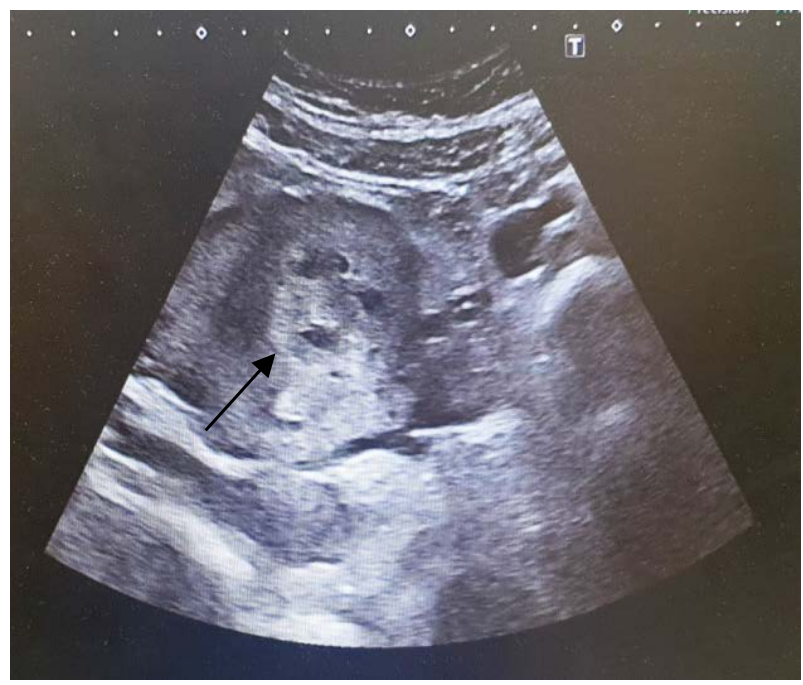

Figure 6. Appearance of necrotic submucosal fibroid or arteriovenous malformation on transparietal ultrasound $(\rightarrow)$. 


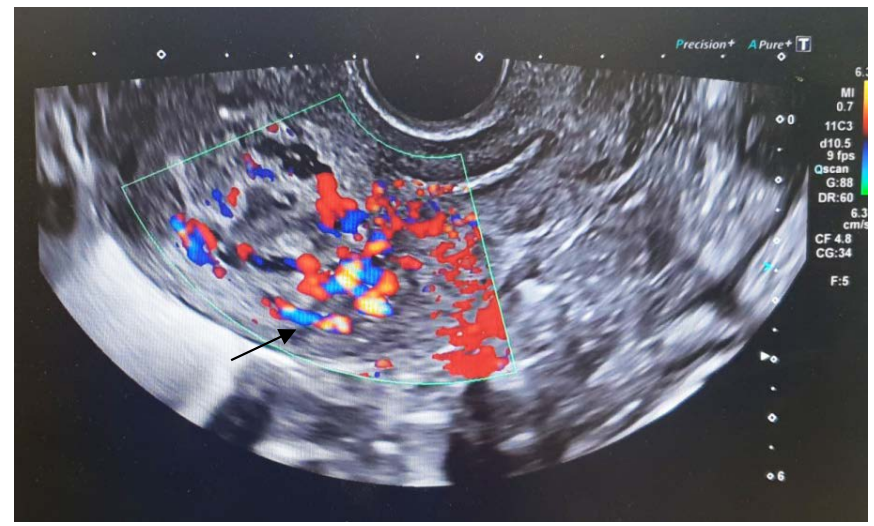

Figure 7. Sonographic appearance of a highly vascularized intrauterine malformation $(\rightarrow)$.

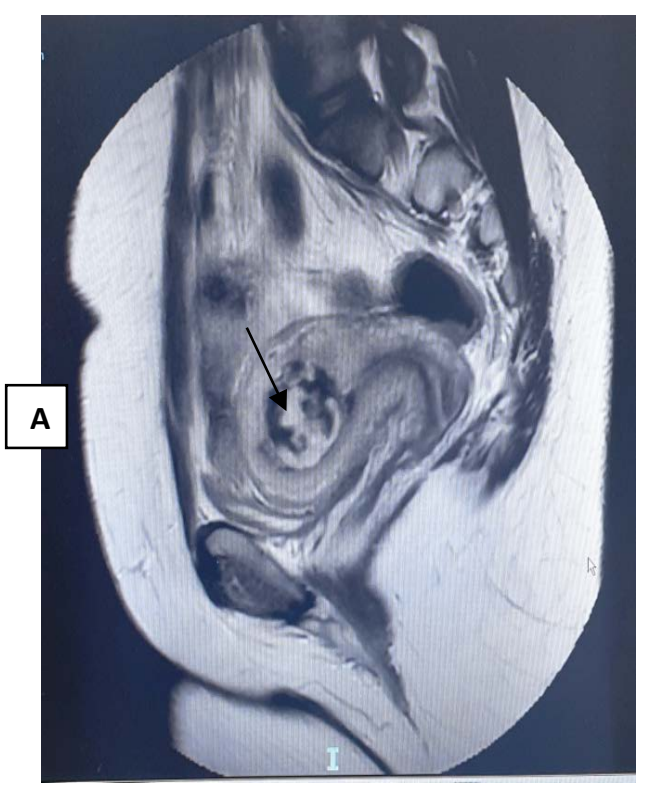

Figure 8. MRI appearance of intrauterine arteriovenous malformation in sagittal section.

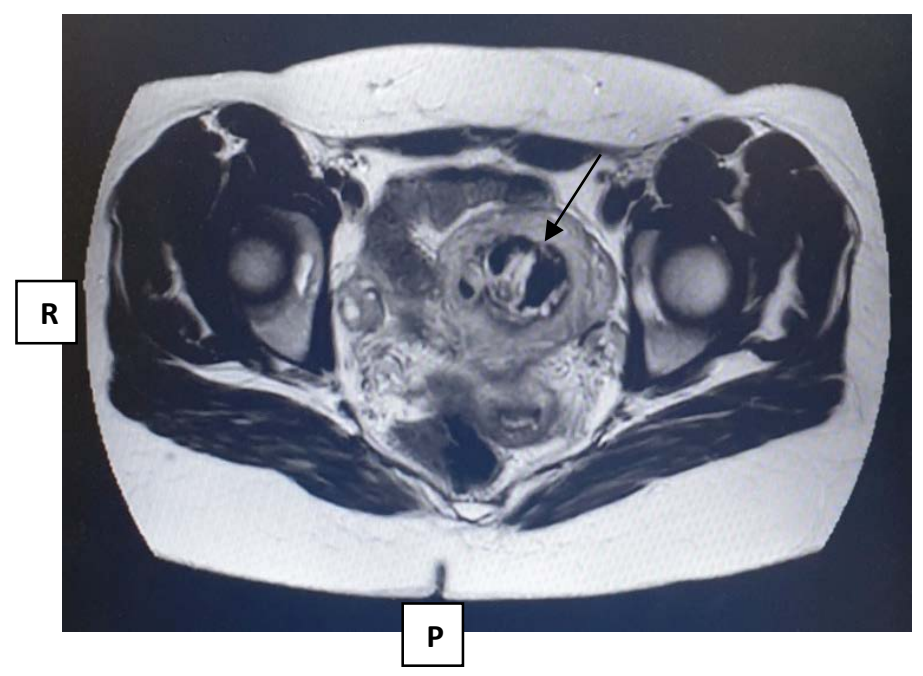

Figure 9. MRI appearance of intrauterine arteriovenous malformation in cross section. 


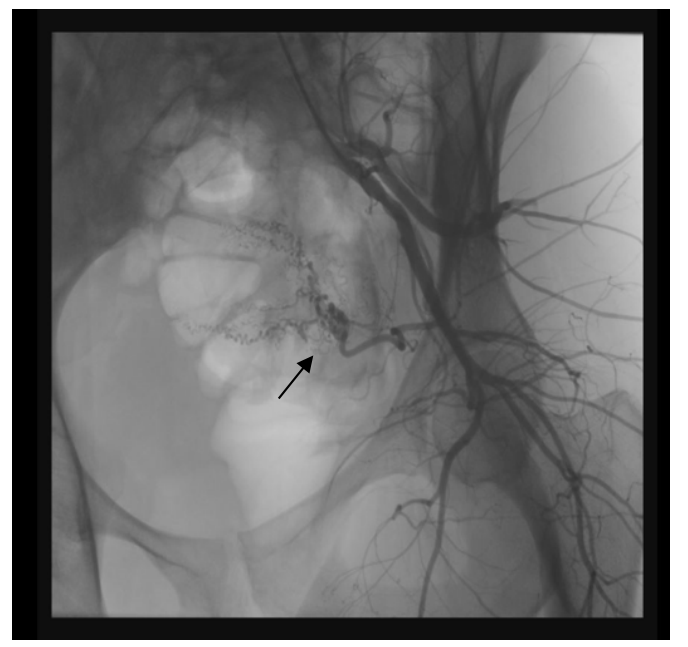

Figure 10. Angiographic sequence showing an UAVM of the left uterine artery $(\rightarrow)$ (Case $\left.\mathrm{n}^{\circ} 2\right)$.

Hospitalization of the patient with prescription of a morphine pump. A diagnostic and operative hysteroscopy is performed within 48 hours: the appearance of placental retention without evident abundant vascularization (Figure 11). Superficial retention resection and endometrectomy. Anatomopathological examination described foci of adenomyosis. The patient returns to a regular period after five months. Very poor follow-up experience by the patient who developed a depressive syndrome with nightmares every night.

\section{Case $\mathrm{n}^{\circ} 3$}

Mrs CHE, 43 years old, G4P2 (2 vaginal deliveries, 1 spontaneous miscarriage, 1 clear egg at $7 \mathrm{WG}+5$ days) presented trophoblastic retention with suspicion of UAVM following treatment of the clear egg. In fact, on day 10 after administration of MISOPROSTOL, the ultrasound found an intrauterine image of $8 \mathrm{~mm}$. A month later, we find a hematometry slide with an intracavitary image of $12 \mathrm{~mm}$. During the ultrasound examination 15 days later, a $17 \mathrm{~mm}$ image appeared, taking intensely the Doppler from the fundus, suggestive of a UAVM (Figure 12 \& Figure 13).

The MRI found an intrauterine formation of $2 \mathrm{~cm}$ suggesting in the first place UAVM (Figure 14 \& Figure 15). Faced with the strong suspicion of UAVM, embolization of the right uterine artery is performed preoperatively. Angiography shows an opacification of an enlarged vascular network without early venous return, in favor of a trophoblastic residue (Figure 16). Embolization is performed via the right uterine artery in free flow beyond the cervicovaginal branches with GELITASPON, until vascular stasis. A final check in the left uterine artery fails to recover. The same hysteroscopic embolization sequence is carried out as for case $n^{\circ} 2$.

Diagnostic hysteroscopy finds heterogeneous intrauterine formation at the level of the right anterolateral wall of the uterus with images of vascular lattices, without bleeding on contact (Figure 17). Loop resection is performed initially without current and then with bipolar current of the entire lesion. 


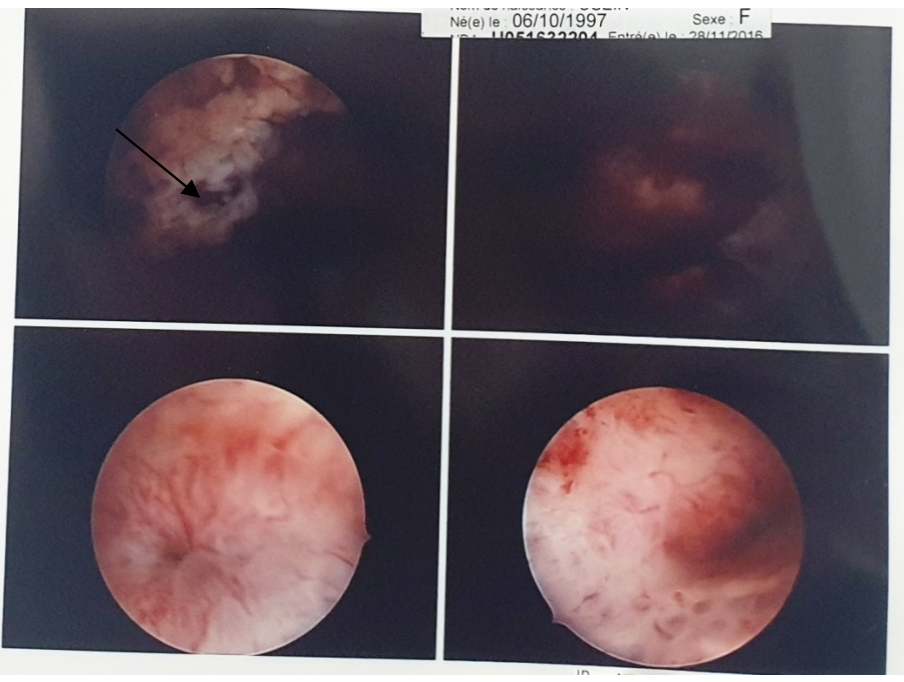

Figure 11. Hysteroscopic appearance of necrotic retained placenta $(\rightarrow)\left(\right.$ Case $\left.n^{\circ} 2\right)$.

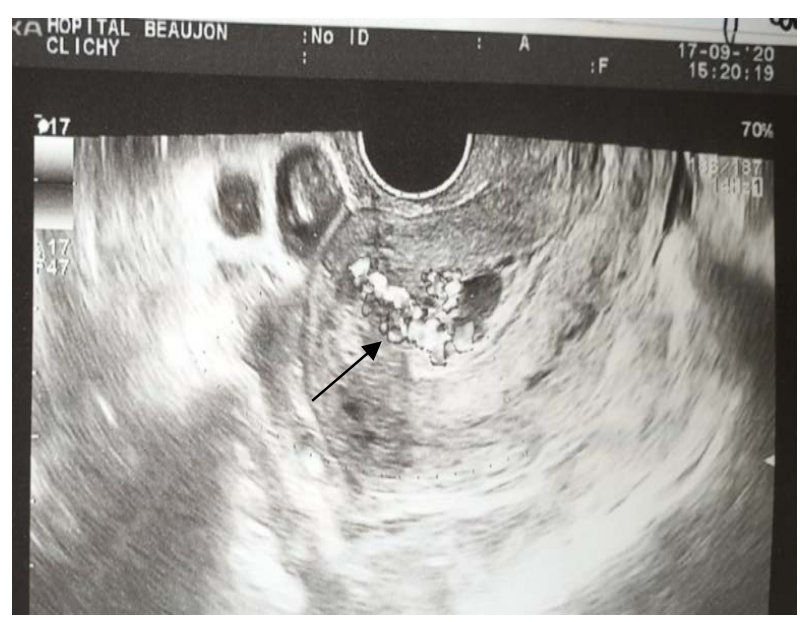

Figure 12. Ultrasound image taken in the emergency with suspicion of UAVM $(\rightarrow)$ (Case $\left.\mathrm{n}^{\circ} 3\right)$.

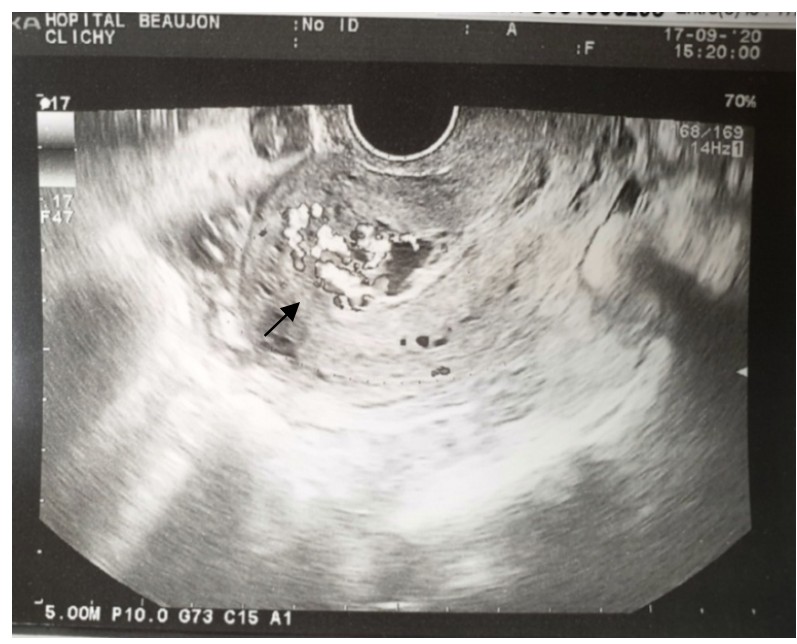

Figure 13. Ultrasound image of UAVM intensely enhancing with $17 \mathrm{~mm}$ color Doppler at the fundal level $(\rightarrow)\left(\right.$ Case $\left.n^{\circ} 3\right)$. 


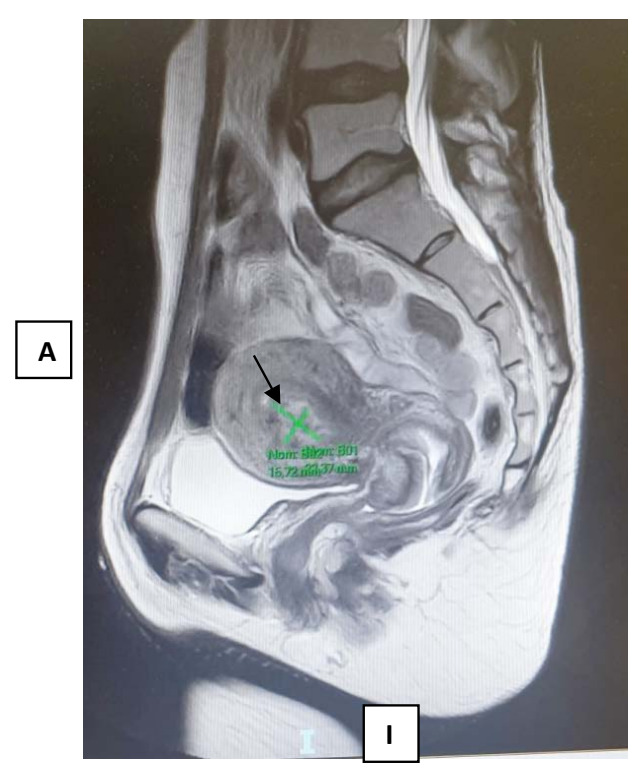

Figure 14. MRI image in sagittal sectionshowing an aspect of UAVM $(\rightarrow)$.

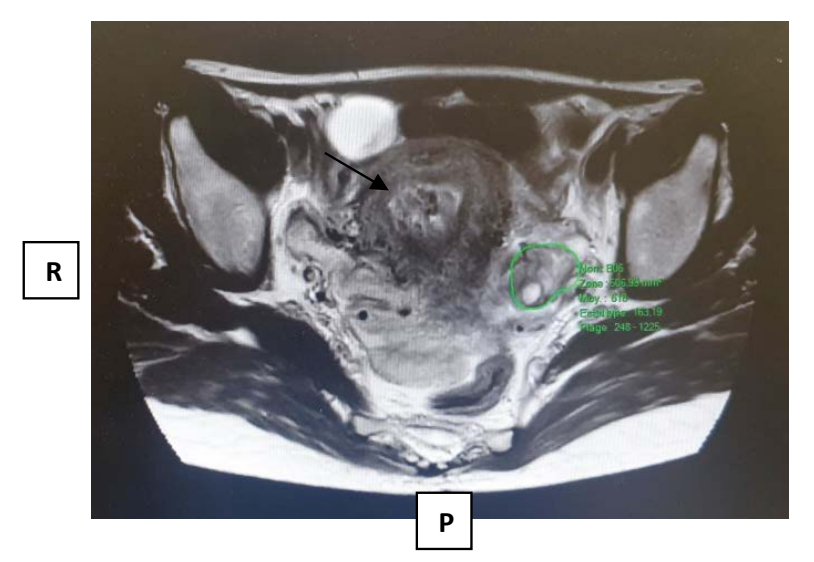

Figure 15. Cross sectional MRI showing an aspect of UAVM $(\rightarrow)$.

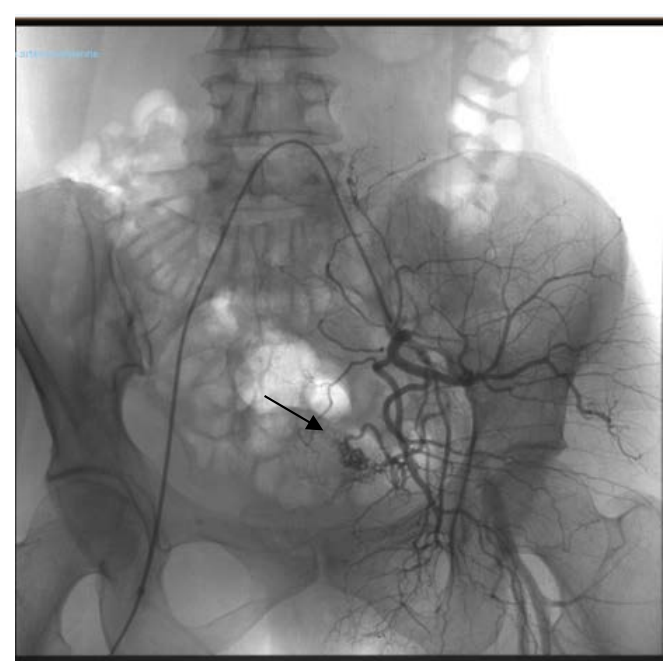

Figure 16. Angiography sequence revealing an UAVM of the right uterine artery $(\rightarrow)$ $\left(\right.$ Case $\left.n^{\circ} 3\right)$. 


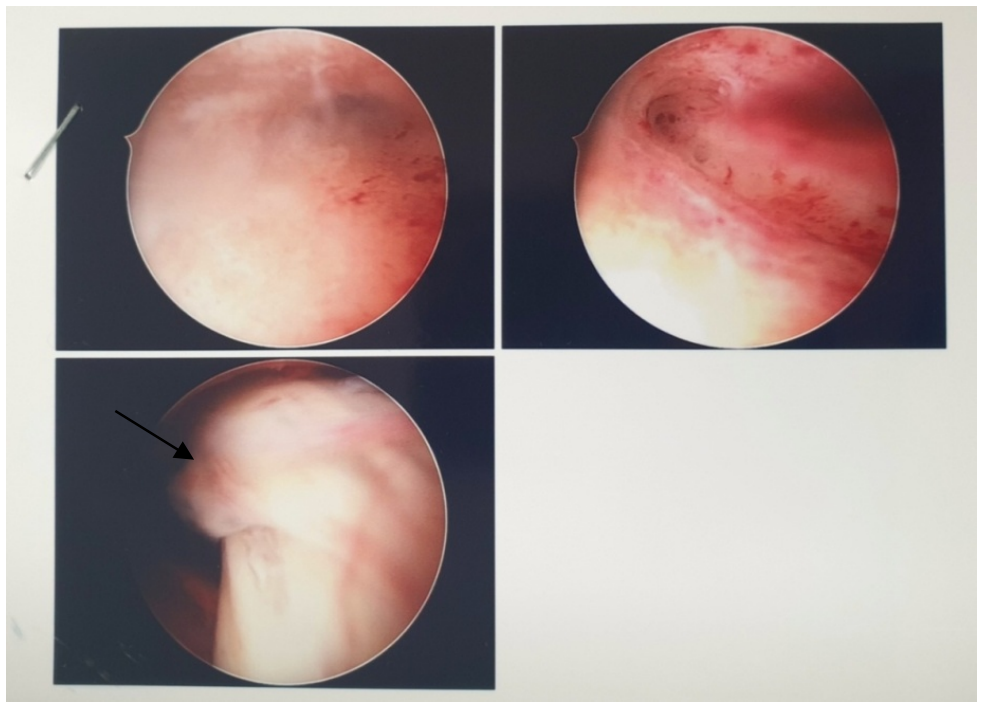

Figure 17. Images of UAVM at the level of the right anterolateral wall on hysteroscopy $(\rightarrow)\left(\right.$ Case $\left.^{\circ} 3\right)$.

Anatomopathological study: placental debris with significant signs of retention.

\section{Discussion Section}

For better visibility, we have divided our review of the literature into several parts concerning the definition, diagnosis and management.

\section{Definition and pathophysiology}

UAVM can be defined as a structural abnormality involving abnormal communication between arteries and veins that bypass the capillary system. They are characterized by the presence of a high pressure gradient across the arterial and venous system which allows high vascular flow through the nidus, the intermediate network of vessels communicating from the arteries to the veins [1]. UAVMs are divided into congenital and acquired. Congenital UAVMs result from a defect in differentiation of the primary capillary plexus during fetal angiogenesis [1]. They tend to extend beyond the margins of the uterus into the pelvis [2]. In contrast, so-called acquired UAVMs occur after damage to uterine tissue. These acquired UAVMs may result from: A miscarriage or voluntary termination of pregnancy (dilation and curettage), cesarean section [3], placement of an intrauterine device, a uterine revision, carcinoma of the cervix or endometrium, uterine infection, trophoblastic disease, and endometriosis are some of the possible but rare causes of UAVM [4]. Finally, cases of post coital metrorrhagia linked to cervical UAVM have also been described in the context of Distilbene syndrome [4].

The most plausible pathophysiological hypothesis is that of a traumatic lesion having ruptured or weakened the wall of an intramural artery and of a vein of the adjacent myometrial plexus and whose scarring would have induced the formation of a fistula between these two, vascular structures [5]. Pregnancy 
seems to play a preponderant role in the pathogenesis of some of these UAVM by connecting arterial and myometrial venous vessels during necrosis of the chorio-placental villi [6]. Associated hormonal changes, such as elevated human chorionic gonadotropin (HCG), may play a role in the proliferation of UAVM through a still unclear mechanism. Likewise, women undergoing fertility treatments may have high estrogen levels which could cause endothelial proliferation and endometrial differentiation [1]. Histological examination reveals several small arteriovenous fistulas, between the intramural arterial branches and the myometrial venous plexus [2].

Rare or underdiagnosed pathology?

At the uterine level, the frequency of UAVMs is low, although their actual incidence is currently unknown. Due to their extreme variability in size and topography, their clinical expression is unpredictable [6]. About a hundred cases have been reported in the literature. However, reported cases are increasing due to the increasing availability of imaging modalities such as ultrasound, computed tomography (CT) and magnetic resonance imaging (MRI) [2]. The first diagnosis to be evoked in the face of post-abortion metrorrhagia (spontaneous or provoked) remains the retention of ovular material. However, failure to diagnose UAVM prior to aspiration may be responsible for particularly severe life-threatening bleeding. Indeed, in the event of acquired MVUA, these procedures can create a wound in the vessels and lead to more or less abundant bleeding depending on the extent of the arterial flow and the vascular lesions [5].

Clinical presentation

With various sizes and locations, UAVM cause a variety of clinical manifestations [3]. The most common symptom is heavy bleeding (menorrhagia or metrorrhagia) that is resistant to medical treatment [6]. Patients may also present with anemia and hypotension. According to the literature, approximately $40 \%$ of patients with UAVM required a blood transfusion. Pelvic discomfort, urinary symptoms (polyuria and incontinence) and dyspareunia have also been reported. Congestive heart failure secondary to the detour of blood into the venous system is possible but rare. Finally, patients may also remain asymptomatic [1].

The average age at diagnosis is 30 years [1]. Acquired UAVMs are usually identified in multiparous women of childbearing age. As mentioned above, some acquired MAUVs are diagnosed after surgery on suspected retained ovarian material. If there is heavy bleeding on aspiration, uterine AVM should be suspected and appropriate precautions taken [1]. The literature also reports hemostatic hysterectomies after hemostatic curettage without the etiology of the bleeding being found [5]. It is likely that some of these hysterectomies are related to UAVMs.

\section{Physical examination and paraclinical diagnosis}

The clinical examination does not usually reveal any particular warning sign. Some authors suggest that a throbbing mass may be seen on vaginal examination. There is currently no consensus on the diagnostic criteria for imaging-acquired UAVM. Ultrasound, angiography, hysterosalpingography, hyste- 
roscopy, CT scan and MRI have been described in the literature as diagnostic methods [5].

Pelvic ultrasound

Ultrasound, which is inexpensive and noninvasive, plays a predominant role in the diagnosis of UAVM. The diagnosis is based on the presence of hypoechoic areas with irregular contours in the myometrium, with turbulent flow on color Doppler sometimes responsible for aliasing. Color Doppler allows early identification of a vascular anomaly that has gone unnoticed in B mode and increases the sensitivity of the ultrasound. Arterial velocity is high and resistance is low [4]. Three-dimensional ultrasound coupled with Doppler can also be useful, allowing image reconstruction and flow quantification [4]. Ultrasound can visualize the lesion and influence the therapeutic options [6]. In case of doubt, it seems relevant to propose, as a second line, an angio-MRI.

\section{Pelvic MRI}

MRI is a determining factor in the diagnosis of UAVM, even if it is not yet systematically performed. It is of twofold interest. On the one hand, it makes it possible to eliminate other diagnoses such as adenomyosis [1]. On the other hand, it allows a precise geographical localization of the malformation by visualizing the feeding vessels. This type of malformation presents as an intramyometrial serpiginous mass leading to a zone of diffuse or localized rupture at the junction zone, called "signal void". On T2 weighting, the lesions are homogeneous and in hyper signal [3]. The main disadvantages of MRI are its high cost, its limited availability in some countries and its relatively long acquisition time [2].

Angiography

Angiography is the gold standard. Angiographic analysis can reveal an enlarged, tortuous uterine artery with a large feeder vessel and early venous return during the arterial phase [2]. It allows to differentiate UAVM from other vascular tumors, and to guide therapeutic embolization. It therefore allows confirmation of the diagnosis and is the first step in endovascular treatment [3]. As it is an invasive procedure, it should be reserved for therapeutic management [5]. It can be used alone or prior to surgery [6]. CT angiography is preferred in unstable patients with significant bleeding [2].

\section{Hysteroscopy}

The use of hysteroscopy has been reported. It is of limited value in the context of active hemorrhage. Visualization of the UAVM as a pulsatile, bumpy vascular structure on the surface of the uterine cavity has been described [4]. Hysteroscopy has also been used to diagnose UAVM, but its value is limited. However, it may be useful for UAVM located just below the endometrium [2]. Recently, a particular entity of retained product of conception (RPOC) called "Marked Vascularization" or "Highly Vascularized" has been described with an estimated prevalence of $18 \%$ based on Doppler findings, and frequently associated with UAVM [7]. In this context, the question arises of diagnostic hysteroscopy to visualize the presence of ovarian retention given the frequency of occurrence of post abortion UAVM. In addition, the cost of hysteroscopy remains lower than 
that of angiography to rule out a differential or associated diagnosis.

\section{Treatment}

There is no clear consensus on the best treatment for UAVMs. Current medical and surgical options are based on clinician experience and published case reports. Treatment depends on the extent of the bleeding [8].

\section{Therapeutic abstention or medical treatment}

In a stable condition, treatment modalities will depend on the technical possibilities of the care center and the patient's wishes. In the case of a desire for pregnancy or uterine preservation, as well as in the case of asymptomatic acquired AVM discovered incidentally, abstention from treatment may be indicated in the presence of a lesion likely to regress spontaneously [6]. Medical treatments based on methylergonovine maleate, danazol and $\mathrm{GnRH}$ agonists have been described [8]. They are reported to be associated with regression of the lesions on ultrasound [2].

\section{Interventional radiology: Embolization}

If conservative management is ineffective, the recommended treatment is embolization of the uterine artery or internal iliac artery, or even selective embolization of the UAVM focus [8]. Embolization allows both confirmation of the pretherapeutic diagnosis (arteriographic phase) and rapid treatment of the patient [5]. It is usually performed under local anesthesia, using a catheter placed in the right or left femoral artery, which allows selective embolization of the uterine arteries. The final success rate is between $71 \%$ and $93 \%$, and iterative embolization after failure of the first attempt is performed in $32 \%$ to $40 \%$ of cases [ 4 ]. Selective embolization can be uni or bilateral depending on the topography of the feeding arteries [5]. It allows preservation of ovarian function, as well as fertility [7]. A particle size (commercial size) greater than $700 \mu \mathrm{m}$ has been chosen to avoid both migration through arteriovenous shunts and the risk of uterine necrosis, which has been reported with diameters less than $500 \mu \mathrm{m}$ [7]. The use of particles larger than $500 \mu \mathrm{m}$ should therefore be preferred in order to avoid involuntary embolization of the utero-ovarian anastomosis and thus avoid the risk of ovarian ischemia [4].

There is often a combination of non-absorbable and absorbable particles. This combination allows, thanks to the microcoils, obturation of the supramilimetric vessels while preserving the myometrial microvascularization, and thanks to the resorbable material, an immediate seal [4].

The main complications reported are the following:

- Post-embolization syndrome with massive necrosis of the uterine infarct.

- Transient or permanent amenorrhea and subfertility. The pregnancy rate after arterial embolization treatment varies between $17.4 \%$ and $27 \%$ according to the studies.

- Post-embolization pain requiring morphine derivatives.

- Transient claudication of the buttock and lower limbs.

- Rarely, utero-vaginal and recto-vesico-vaginal fistulas and bladder necrosis are described [2]. These complications are most often related to embolization 
of the internal iliac artery [4].

Post-embolization pregnancies may be marked by the occurrence of spontaneous abortions, placenta previa or accreta, postpartum hemorrhage, and a higher rate of cesarean delivery than in the general population [8].

\section{Surgical treatment}

En cas de saignement cataclysmique menaçant le pronostic vital ou en cas d'échec de l'embolisation après plusieurs tentatives, une hystérectomie hémostatique est indiquée. Ce geste peut également être proposé en cas de récidive de l'hémorragie et de désir de la patiente [6]. Cette solution chirurgicale a longtemps été considérée comme le traitement de choix de la UAVM acquise symptomatique [5]. La ligature utérine laparoscopique a été rapportée comme une alternative minimale. La laparotomie reste une procédure invasive, et les risques associés à l'anesthésie générale ne sont pas anodins [8]. Cependant, dans les hôpitaux et dans les pays qui ne disposent pas de radiologie interventionnelle, les différentes techniques chirurgicales mini-invasives peuvent être entreprises face à des signes d'instabilité hémodynamique ou de choc hémorragique [5]. Le traitement par coagulation laparoscopique des vaisseaux utérins à l'aide de pinces bipolaires a été rapporté avec succès. Les auteurs ont démontré la diminution manifeste du débit de l'UAVM en Doppler pulsé [3]. Une combinaison d'embolisation et de résection laparoscopique des lésions a également été décrite [2]. La pose d'une sonde de Foley intra-utérine peut être une bonne alternative en cas de saignement foudroyant [2].

\section{The Place of Hysteroscopy}

In the study by Calzolari et al. [8], hysteroscopy was found to be a feasible and safe alternative treatment modality for MAUV. Thus, all cases were successfully treated as outpatients in a single step of surgical hysteroscopy, without recurrence during the follow-up period. The patients had no complications during the procedure and throughout the follow-up. The median duration of the procedure was 30 minutes, which is significantly less than the 60 minutes required for arterial embolization treatment. This is due to the better exposure of the uterine cavity obtained throughout the hysteroscopy, as opposed to the indirect approach with embolization. All patients were declared fit for discharge the same day, whereas embolization required hospitalization. In addition, after the procedure, no prescription of analgesics was necessary. More than half of the patients obtained a pregnancy after hysteroscopy with an average delay of 6 months and one third of them was over 40 years old or did not want a new pregnancy. In the literature, $27 \%$ of patients had a pregnancy after embolization treatment, with a delay of 15 months. No recurrence of MAUV was noted during follow-up between 4 and 30 months with a median of 24 months [8]. Finally, during resection of a retention, it is sometimes possible to find the presence of a uterine fibroid or an adenomyosis nodule, which could therefore be at the origin of this retention. Resection of this formation on which the retention is inserted 
can then be carried out during the same operation.

The diagnosis of MAUV is under-diagnosed, particularly in the aftermath of abortion. Thus, by evoking more often the hypothesis of a UAVM, we report 3 cases that occurred within one year. The diagnosis of FMU was not evoked for the first case, but it allowed a systematic search for this pathology. The majority of VMU are the result of trophoblastic retention. Our case series confirms this hypothesis. In all 3 cases, the VSU developed after aspiration or miscarriage. The average time from fetal loss to diagnosis was 4 to 5 weeks. This is consistent with what is described in the literature. In our department, all our patients had ultrasound coupled with color Doppler. For the first patient, a diagnostic and then operative hysteroscopy was carried out straight away, which led, on the one hand, to abundant haemorrhage with placement of an endo-uterine probe and a stay in intensive care, and on the other hand, to the absence of effective treatment of the UAVM which persisted for several months. Thus, for our other two patients, in view of the suspicion of AVMU on pelvic MRI, a first embolization was performed before a hysteroscopic procedure 24 to 48 hours later. Under these conditions, the hysteroscopies performed allowed complete resection without hemorrhage of the AVMU residues and restoration of an unharmed uterine cavity. Moreover, in one of our cases, we were able to demonstrate foci of adenomyosis.

Although our number of cases is very low, the procedure combining embolization and then hysteroscopy seems to provide several advantages:

- Resection of the AVMU under optimal conditions with reduced bleeding risk.

- Much shorter healing and monitoring time than embolization alone with a reduction in the number of patients lost to follow-up.

- Optimal evaluation of the uterine cavity after treatment.

- Possibility of applying an anti-adhesive gel.

\section{Conclusion}

UAVM are rare but should be suspected in the presence of repeated abnormal bleeding in young women with a history of fetal loss or endometrial intervention. This would avoid hemostatic curettage which would be both unnecessary and harmful in this context. Their diagnosis is based on ultrasound. Arteriography remains a reference examination in severe cases because it allows both diagnosis and treatment. Nowadays, hysterectomy tends to become the emergency intervention for extreme cases refractory to other therapies. Based on our experience with three cases, we propose a new therapeutic alternative combining interventional radiology and surgery. The potential benefits of the embolization-hysteroscopy sequence need to be evaluated with high power studies.

\section{Consent}

Patients gave their consent to participate in the study. 


\section{Conflicts of Interest}

The authors declare no conflicts of interest regarding the publication of this paper.

\section{References}

[1] Yoon, D.J., Jones, M., Al Taani, J., Buhimschi, C. and Dowell, J.D. (2016) A Systematic Review of Acquired Uterine Arteriovenous Malformations: Pathophysiology, Diagnosis, and Transcatheter Treatment. American Journal of Perinatology Reports, 6, e6-e14. https://doi.org/10.1055/s-0035-1563721

[2] Szpera-Goździewicz, A., Gruca-Stryjak, K., Bręborowicz, G.H. and Ropacka-Lesiak, M. (2018) Uterine Arteriovenous Malformation: Diagnosis and Management. Ginekologia Polska, 89, 276-279. https://doi.org/10.5603/GP.a2018.0047

[3] Rubod, C., Mubiayia, N., Robert, Y. and Vinatier, D. (2005) Arteriovenous Malformation. A Rare Cause of Recurrent Metrorrhagia. Gynécologie Obstétrique \& Fertilité, 33, 511-513. http://france.elsevier.com/direct/GYOBFE/Uterine https://doi.org/10.1016/j.gyobfe.2005.05.018

[4] Sanguin, S., Lanta-Delmas, S., Le Blanche, A., Grardel-Chambenoit, E., Merviel, P., Gondry, J. and Fauvet, R. (2011) Uterine Arteriovenous Malformations: Diagnosis and Treatment in 2011. Gynécologie Obstétrique \& Fertilité, 39, 722-727. https://doi.org/10.1016/j.gyobfe.2011.08.013

[5] Rabarikoto, H.F., Rabetsimamanga, L.A.Z., Randriamahavonjy, R., Goudy, G., Secourgeon, J.F., Vignelongue, M.H., Higue, D., Andrianampanalinarivo, H.R. and Brun, J.L. (2013) Uterine Arteriovenous Malformations (Report of Six Cases). Revue D’anesthésie-réanimation et de Médecine D’ urgence, 5, 5-10.

http://www.rarmu.com/

[6] Delotte, J., Chevalier, P., Desprez, B., Castillon, J.M. and Bongain, A. (2004) A Rare Diagnosis of Uterine Haemorrage: Uterine Arteriovenous Malformation. La Lettre du Gynécologue, n² 290, 31-33.

[7] Paisant-Thouveny, P.B.F., Yahya, S., Bouvier, A., Nedelcu, C., Boussion, F., Sentilhes, L., Willoteaux, S. and Aube, C. (2016) Uterine Artery Embolization for Retained Products of Conception with Marked Vascularity: A Safe and Efficient First-Line Treatment. Cardio Vascular and Interventional Radiology, 40, 520-529. https://doi.org/10.1007/s00270-016-1543-7

[8] Calzolari, S., Cozzolino, M., Castellacci, E., Dubini, V., Farruggia, A. and Sisti, G. (2017) Hysteroscopic Management of Uterine Arteriovenous Malformation. Journal of the Society of Laparoendoscopic Surgeons 21, e2016.00109.

https://doi.org/10.4293/JSLS.2016.00109 